\title{
Influence of Microbial Growth on Hydraulic Properties of Pore Networks
}

\author{
MARTIN THULLNER $^{1, \star}$, JOSEF ZEYER ${ }^{1}$ and WOLFGANG \\ KINZELBACH ${ }^{2}$ \\ ${ }^{1}$ Institute of Terrestrial Ecology, Swiss Federal Institute of Technology (ETH) Zurich, Switzerland \\ ${ }^{2}$ Institute of Hydromechanics and Water Resources Management, Swiss Federal Institute \\ of Technology (ETH) Zurich, Switzerland
}

(Received: 29 November 2000; in final form: 11 September 2001)

\begin{abstract}
From laboratory experiments it is known that bacterial biomass is able to influence the hydraulic properties of saturated porous media, an effect called bioclogging. To interpret the observations of these experiments and to predict possible bioclogging effects on the field scale it is necessary to use transport models, which are able to include bioclogging. For these models it is necessary to know the relation between the amount of biomass and the hydraulic conductivity of the porous medium. Usually these relations were determined using bundles of parallel pore channels and do not account for interconnections between the pores in more than one dimension. The present study uses two-dimensional pore network models to study the effects of bioclogging on the pore scale. Numerical simulations were done for two different scenarios of the growth of biomass in the pores. Scenario 1 assumes microbial growth in discrete colonies clogging particular pores completely. Scenario 2 assumes microbial growth as a biofilm growing on the wall of each pore. In both scenarios the hydraulic conductivity was reduced by at least two orders of magnitude, but for the colony scenario much less biomass was needed to get a maximal clogging effect and a better agreement with previously published experimental data could be found. For both scenarios it was shown that heterogeneous pore networks could be clogged with less biomass than more homogeneous ones.
\end{abstract}

Key words: bioclogging, hydraulic conductivity, porosity, pore blocking, biofilm, pore scale heterogeneity.

\section{Introduction}

The ability of bacterial biomass to change hydraulic properties of a saturated porous medium has been observed in laboratory studies (Baveye et al., 1998). The possible influence of this phenomenon - called bioclogging - on the applicability of bioremediation techniques can be envisioned in two contrary ways. Bioclogging may reduce the success of bioremediation because contaminated parts of an aquifer can clog due to microbial activity and rates of degradation can decrease (Baveye et al., 1998). On the other hand, controlled clogging of an aquifer may be used to clog preferential paths and get a more homogeneous sweep or to build up

\footnotetext{
^ Author for correspondence: e-mail: thullner@ito.umnw.ethz.ch
} 
biobarriers which could be used to increase the success of remediation (Johnston et al., 1997).

The decrease in hydraulic conductivity due to microbial growth has been investigated in column experiments. Several authors reported a significant reduction of hydraulic conductivity due to bioclogging (Taylor and Jaffé, 1990a; Cunningham et al., 1991; Vandevivere and Baveye, 1992; Brough et al., 1997; Johnston et al., 1997; Wu et al., 1997). While circumstances necessary to clog the test columns varied, Cunningham et al. (1991) used a column inoculated with bacteria and applied a constant head difference between inflow and outflow. A decrease of more than $90 \%$ in hydraulic conductivity and $50-90 \%$ in porosity was observed. Taylor and Jaffé (1990a) used columns with a constant influx. Reduction in hydraulic conductivity by three orders of magnitude was observed along with an increase of biomass. In addition a change in dispersivity was observed. Vandevivere and Baveye (1992) performed similar column tests with varying boundary conditions. They observed a reduction of hydraulic conductivity by three orders of magnitude but only a maximum porosity reduction of less than $10 \%$. In a series of 35 column experiments, Brough et al. (1997) observed a decrease of hydraulic conductivity of between 28 and $79 \%$. It was found that a procedure in which activated sludge microorganisms were added during the filling of the column with sand was the best method to reach the desired high reductions of hydraulic conductivity. Johnston et al. (1997) investigated the production of polysaccharides in aquifer material under different nutrient supply. In a column experiment performed under anaerobic conditions they observed a reduction of hydraulic conductivity by a factor of about 14. Wu et al. (1997) performed column experiments with constant head boundaries. A reduction in hydraulic conductivity of about one order of magnitude was observed but due to gas production this reduction could not be attributed to the biomass alone (Baveye and Dumestre, 1998). In addition to these column studies Dupin and McCarty (2000) and Kim and Fogler (2000) investigated microbial growth and its influence on the hydraulic properties of microscopic pore networks. Both authors observed high reductions in hydraulic conductivities. Dupin and McCarty (2000) could show that the morphology of microbial growth depends on the $\mathrm{pH}$-value, whereas Kim and Fogler (2000) were focusing on the effect of shear forces and nutrient feeding, showing that biofilms may resist in the pores even when nutrient supply has stopped.

Besides bioclogging there are also non biological mechanisms reducing the hydraulic conductivity of a porous medium. Such processes may be, for example, particle deposition as investigated in connection with deep bed filtration (e.g. Tien and Payatakes, 1979), particle deposition in combination with chemically induced processes which is causing formation damage of petroleum reservoirs (e.g. Wojtanowicz et al., 1988; Chang and Civan, 1997) or the capillary instability of wetting films causing pore blocking in gas-condensate systems (e.g. Coskuner, 1997). The mechanisms causing these non biological clogging processes are well 
investigated but differ from the mechanisms causing the clogging of pores due to biomass production.

Several models have been introduced to simulate the observed interactions between biomass and the hydraulic properties of the porous media. Usually these models are based on the assumption of a homogeneous biofilm covering the surface of the grains (e.g. Taylor et al., 1990). A reduction of hydraulic conductivity caused by biofilm growth was theoretically derived assuming bundles of parallel pores. Especially for fine-textured materials these models could not predict observed hydraulic conductivity reductions satisfactorily (Vandevivere, 1995; Vandevivere et al., 1995). In Clement et al. (1996) a clogging model was presented, which made no explicit assumptions on the distribution of the biomass in the pores. The prediction of hydraulic conductivity reductions of the model of Clement et al. (1996) was nearly identical to predictions of Taylor et al. (1990), and thus the criticism of Vandevivere et al. (1995) also applies to the model of Clement et al. (1996). In Vandevivere et al. (1995) it was assumed that the discrepancy between model predictions and experimental results is caused by the fact that microorganisms can also form colonies, which influence the hydraulic conductivity differently than a biofilm. In addition, it was suggested to use pore networks instead of pore bundles in order to account for interpore connections (Loehle and Johnson, 1994; Vandevivere et al., 1995). In Suchomel et al. (1998a) a pore network model was introduced and applied to previously published data (Suchomel et al., 1998b). The model produced realistic results assuming the growth of a biofilm on the walls of cylindrical pores. Kim and Fogler (2000) could also reproduce their experimental data with pore network simulations assuming biofilm growth. In contrast to this Dupin and McCarty (2000) showed that their experimental observations could be explained with network model simulations assuming the growth of biomass in aggregates, whereas assuming a biofilm could not explain their observations.

Until now no theoretically derived hydraulic conductivity versus porosity relations are published, which account for interpore connections in more than one dimension and which account for microbial growth morphologies different to a biofilm. The available relations could not explain experimentally observed clogging effects. Thus the question arises whether approaches, which include a multidimensional pore structure and alternative growth morphologies, yield a better description of hydraulic conductivity reduction due to the biomass.

For the present project we used pore network models as a tool to simulate the change of the hydraulic properties due to microbial growth. The investigation of general properties of such networks is a subject of percolation theory (Stauffer and Aharony, 1992). Results from percolation theory were already used for the description of hydraulic properties of porous media (Berkowitz and Balberg, 1993; Sahimi, 1995). Pore network models were already used successfully for the simulation of non biological clogging processes (e.g. Rege and Fogler, 1987; Imdakm and Sahimi 1991; Rege and Fogler, 1991; Ewing and Gupta, 1994; Burganos et al., 1995; Kaiser, 1997; Wang and Mohanty, 1999; Lee and Koplik, 2001). 
As the existing hydraulic conductivity versus porosity relations were not able to reproduce experimental observations available in literature, we were focusing on the question whether relations derived from pore network simulations were generally able to explain the observed small reductions of porosity already causing a large reduction in hydraulic conductivity. In addition, we wanted to investigate the influence of the morphology of microbial growth on the results of the pore network simulations. For this reason we decided to use two-dimensional pore networks consisting of cylindrical pores as a simplified representation of a porous medium. To investigate the influence of the morphology of microbial growth, we chose two different scenarios describing biomass growth. Following the suggestions made in (Vandevivere et al., 1995) we assumed for the first scenario the biomass to grow as micro-colonies, which plug pores entirely. Generally it would be possible to assume different ways to correlate the size of a pore with its probability of becoming clogged (e.g. preferential clogging of the largest pores, preferential clogging of the smallest pores or no correlation at all). As experimental observations showed that relatively small amounts of biomass could cause high reductions in hydraulic conductivity, we decided to let the micro-colonies plug the smallest pores preferentially, giving the biomass a high clogging efficiency. For natural porous media this scenario corresponds to a preferential plugging of the pore throats, which are the bottle necks for the water flow.

In comparison to this colony scenario, we assumed for the second scenario the biomass to grow as a biofilm on the walls of each pore. For both scenarios we calculated the changes of hydraulic conductivity and pore volume of the entire pore networks to derive hydraulic conductivity versus porosity relations, which could be compared to results published in literature.

\section{Model Description}

\subsection{MODEL GEOMETRY}

The model used in this study consists of cylindrical pores. The pores are connected in a two-dimensional rectangular grid. All pores are assumed to have the same length $l$, the radius $r_{i}$ of the pore $i$ is taken from a lognormal distribution. The sites connecting the pores (in the following referred to as sites) are assumed to have no volume.

\subsection{FLOW MODEL}

The volume $v_{i}$ of a single pore $i$ is given by $r_{i}$ and $l$ following the equation

$$
v_{i}=\pi r_{i}^{2} l .
$$

The flow rate $q_{i}$ in a pore $i$ is given by Hagen-Poiseuilles law

$$
q_{i}=-c_{i} \frac{\Delta h_{i}}{l},
$$


where $\Delta h_{i}$ is the hydraulic head difference between the sites at each end of the pore and $c_{i}$ the hydraulic conductivity given by

$$
c_{i}=\frac{\pi \rho g r_{i}^{4}}{8 \eta},
$$

where $\rho$ is the density, $\eta$ the viscosity of the fluid and $g$ the gravitational acceleration.

As shown by Suchomel et al. (1998a) this formulation together with mass conservation leads to a system of linear equations which can be solved for the hydraulic heads $h^{j}$ at each site $j$ efficiently by a successive overrelaxation (SOR) method. In this study the system of flow equations was solved assuming constant head boundaries at two opposite sides of the grid, while the other two sides were no flow boundaries. With the calculated heads $h^{j}$ it is possible to determine the flow rates $q_{i}$ and the average velocities $s_{i}=q_{i} / \pi r_{i}^{2}$ in each pore.

In addition the hydraulic conductivity $C$ of the entire grid can be calculated in analogy to Darcy's law by the expression

$$
C=\frac{Q L}{A \Delta H},
$$

where $Q$ is the total flow rate through the grid, $\Delta H$ the total hydraulic head difference between the constant head boundaries, $L$ the total distance between these boundaries and $A$ the total cross sectional area of the grid. It can be seen that $C$ is proportional to $Q$ and so relative changes in $C$ can be inferred from relative changes of $Q$.

\subsection{TRANSPORT MODEL}

Using the results of the flow model the system of equations describing the transport of a solute within the pore grid can be derived by calculating the solute mass balances for each pore $i$

$$
\frac{\partial m_{i}}{\partial t}=v_{i} \frac{\partial c_{i}}{\partial t}=q_{i} c^{j}-q_{i} c_{i}
$$

with $m_{i}$ being the solute mass and $c_{i}$ being the solute concentration in the pore $i . c^{j}$ is the concentration of solute entering the pore from site $j$. It can be calculated as the flux weighted average of the concentration in the pores with flux towards site $j$ assuming perfect mixing at the site.

In Equation (5) no diffusive processes are included. This simplification was made because numerical tests showed that the addition of a diffusion term to Equation (5) did not influence the results of the simulation. In particular single realization results simulating clogging due to a biofilm (\$3.2) were identical concerning the reduction of hydraulic conductivity and pore volume of the entire network. 
This leads to the time discretized equation

$$
\frac{\Delta c_{i}}{\Delta t}=q_{i} \frac{\sum_{k} q_{k} c_{k}}{\sum_{k} q_{k}}-q_{i} c_{i}
$$

with $k$ as the index of the pores with flux towards the upgradient site $j$ of pore $i$.

The size of the time step is determined by the equation

$$
\Delta t=\frac{l}{v_{\max }},
$$

where $v_{\max }$ is the maximum of the average pore velocities $v_{i}$.

This system of linear equations was solved with an explicit solver.

\subsection{INCORPORATION OF BIOLOGICAL GROWTH}

For this study two different types of microbial growth were incorporated into the model. Like other authors (Taylor and Jaffé, 1990b; Clement et al., 1996; Suchomel et al., 1998a) we did not divide the biomass into bacteria and extracellular polymers (EPS) but regarded them as a unit for both scenarios.

\subsubsection{Microbial Growth in Colonies}

For this scenario the growth of the microorganisms is not coupled to the concentration of a nutrient in the pores but to the radius of the pores. It is assumed that growth occurs in the smallest pores first. Pores in which growth occurs are assumed to be completely filled with biomass whereas all other pores are assumed to be void of biomass. Thus such a network represents a bond percolation model (Stauffer and Aharony, 1992). In practice the presence of a certain amount of biomass in the network is simulated by selecting a number of pores to be filled with biomass and consecutively removing the smallest pores from the grid until a pre-selected volume of biomass is reached. Here it is implicitly assumed that nutrient conditions allow the production of this amount of biomass. The case that the biomass production would be limited by the presence of a nutrient would result in an upper limit for total biomass in the pore network.

\subsubsection{Microbial Growth in a Biofilm}

In this scenario it was assumed that the microorganisms were growing in a biofilm covering the wall of each pore and, therefore, reducing the initial radius. The microbial growth was coupled to the presence of a growth limiting solute nutrient. Assuming that only the amount of nutrient within a limited distance $\delta=\operatorname{exch} \Delta t$ to the surface of the biofilm is available for the microorganisms the mass $m_{i_{\text {bio }}}$ of nutrient available for the microorganisms in pore $i$ within the time step $\Delta t$ is

$$
m_{i_{\mathrm{bio}}}=c_{i} l \pi\left(r_{i}^{2}-\left(r_{i}-\operatorname{exch} \Delta t\right)^{2}\right),
$$

where exch is the exchange parameter. 
The net change of biomass is described by the following equation:

$$
\frac{\Delta b_{i}}{\Delta t}=\frac{\Delta b_{i}^{+}}{\Delta t}-\frac{\Delta b_{i}^{-}}{\Delta t}-\frac{\Delta b_{i}^{\text {shear }}}{\Delta t}=b_{i} \mu^{+}-b_{i} \mu^{-}-s_{i} z 2 \pi r_{i} l
$$

with $b_{i}$ as the biomass and $\mu^{+}$and $\mu^{-}$as the first order rate constants for microbial growth and decay. The nutrient consumption $\Delta m_{i}$ caused by the microbial growth $\Delta b_{i}^{+}$is linked to the change in biomass by $\Delta m_{i}=\Delta b_{i}^{+} / Y$ with $Y$ as a yield factor. $\Delta b_{i}^{+}$is limited by the assumption that $\Delta m_{i}$ should not exceed $m_{i_{\text {bio }}}$. Thus in case $\Delta b_{i}^{+}=\Delta m_{i} Y$ would become larger than $m_{i_{\text {bio }}} Y$, bacterial growth is expressed by a zeroth order rate of $m_{i_{\text {bio }}} Y / \Delta t$ instead of the first order rate $b_{i} \mu^{+}$. This description of microbial growth is a slight simplification of the Monod kinetic (Monod, 1942), which is widely used for the simulation of microbial growth (e.g. Widdowson et al., 1988; Kindred and Celia 1989; MacQuarrie et al., 1990; Chiang et al., 1991; Kinzelbach et al., 1991; Brun et al., 1994; Lensing et al., 1994; Schäfer et al., 1998; Suchomel et al., 1998a). As also done by most of these authors, bacterial decay is described by a first order expression. The term $\Delta b_{i}^{\text {shear }}$ depending on the average velocity $s_{i}$ and the surface of the biofilm in the pore describes the detachment of biomass due to shear forces with $z$ as a rate constant.

By giving the biomass a constant density $d$ (in analogy to Characklis and Marshall (1990) expressed as dry mass per wet volume) it was possible to calculate its volume $v_{i \text { bio }}$ and the resulting change of the radius $r_{i}$ of each pore. Although Characklis and Marshall (1990) reported on an increase of biomass density by a factor of three along the thickness of a biofilm, this effect is not considered in the model, because the typical biofilm thickness is in the range of the pore radii, which are about one order of magnitude smaller than the biofilm thickness presented in Characklis and Marshall (1990). If the average biomass density did increase with an increasing biofilm thickness, the pore radii reduction due to biofilm growth would be overestimated when assuming a constant biomass density.

\subsection{COUPLING OF FLOW, TRANSPORT AND MICROBIAL GROWTH}

\subsubsection{Microbial Growth in Colonies}

As microbial growth was not coupled to specific nutrient concentration in each pore, it was not necessary to include transport calculations for this scenario. The influence of a certain amount of biomass on the hydraulic conductivity of a given pore network was calculated using a three step procedure.

1. The initial hydraulic conductivity $C_{\text {init }}$ was calculated for the entire network assuming no biomass was present. The initial pore volume of the entire grid $V_{\text {init }}$ was determined by integration of the single pore volumes $v_{i}$.

2. A certain number of pores were removed from the grid. Smallest pores were removed first.

3. The hydraulic conductivity $C_{\text {reduced }}$ of the remaining percolation cluster was determined. If no percolation cluster was found, which means that no flow 
path between the inflow and outflow boundary existed, the hydraulic conductivity was set to $C_{\text {reduced }}=0$. The volume of the pores clogged by the biomass $V_{\text {bio }}$ and the volume of the remaining unclogged pores $V_{\text {reduced }}$ was calculated (note that $V_{\text {reduced }}+V_{\text {bio }}=V_{\text {init }}$ ).

Each of these procedures returned a single pair of values: the relative hydraulic conductivity $C_{\text {rel }}=C_{\text {reduced }} / C_{\text {init }}$ and the relative pore space $V_{\text {rel }}=V_{\text {reduced }} / V_{\text {init }}$.

\subsubsection{Microbial Growth in a Biofilm}

For this scenario the simulation of flow, transport and microbial growth had to be coupled. A complete simulation step consisted of the following substeps.

1. The flow problem was solved according to the actual pore radii of each pore.

2. Based on the results of the flow simulation the transport problem was solved.

3. Using the results of substeps 1 and 2 biofilm growth in each pore and thus the changes of the pore radii were calculated.

This results from substep 3 were then used for the next simulation step to calculate the solution of substep 1 and 2. To increase calculation efficiency an option was implemented into the model, which allowed to skip substep 1 of a simulation step. For such simulation steps, substeps 2 and 3 were using the flow solution from the last simulation step, which included substep 1 . The changes in the hydraulic conductivity and the pore space of the entire grid were calculated after each simulation of substep 1.

\section{Simulation of Microbial Clogging Scenarios}

\subsection{SCENARIO 1: CLOGGING DUE TO MICROBIAL COLONIES}

\subsubsection{Numerical Simulations of Hydraulic Conductivity Reduction}

To investigate the influence of microbial colonies on the hydraulic conductivity of the pore networks, simulations were done for $50 \times 50$ pore networks. The log of the radius of each pore was determined randomly, with random numbers following a normal probability density function. The resulting lognormal pore radii distribution had an average or mean dimensionless radius of 1 . For standard deviations we used $\sigma=1 / 3,1 / 2$ and $2 / 3$. These $\sigma$-values are in the range of values typical for sand packs with different heterogeneities (Brutsaert, 1966; Imdakm and Sahimi, 1991; Sugita et al., 1995). For each $\sigma$ a set of 5000 realizations was created. For each of these realizations a volume of biomass was selected randomly (with random numbers equally distributed between 0 and $V_{\text {init }}$ ) and - as described above - a number of pores was removed from the grid and the change in hydraulic conductivity and pore volume was calculated.

Afterwards the range of $V_{\text {rel }}$ for which nonzero $C_{\text {rel }}$-values could be observed was divided into 100 equal intervals. For each of these intervals the $C_{\text {rel-values }}$ were averaged to reduce statistical effects. 


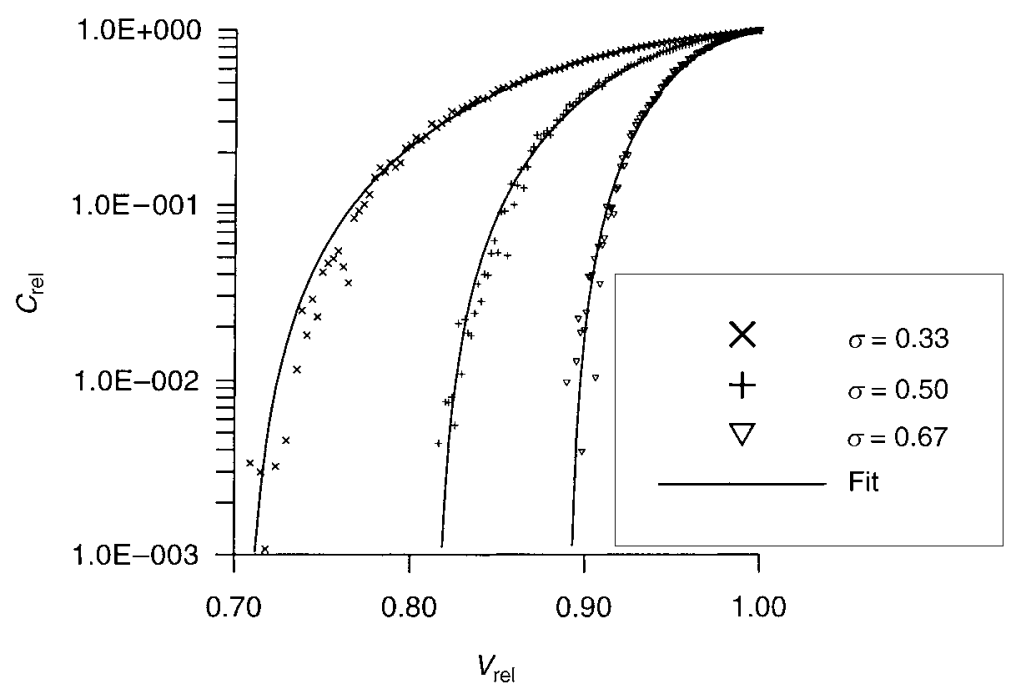

Figure 1. Reduction of hydraulic conductivity due to clogging of pores by microbial colonies.

Figure 1 shows the reduction of hydraulic conductivity due to the pore clogging for $\sigma=1 / 3,1 / 2$ and 2/3. For all investigated values of $\sigma$ a reduction of hydraulic conductivity by more than two orders of magnitude could be observed. The pore volume, for which the maximum reduction of hydraulic conductivity could be achieved, strongly depended on the value of $\sigma$. The higher the heterogeneity the less biomass was needed to reduce the hydraulic conductivity to a given degree.

To express the numerical data with an analytical function we could not use a power law, as done typically to express changes of hydraulic conductivity of pore networks (Sahimi, 1995). The numerical data exhibited a behaviour similar to a power law for small values of hydraulic conductivity, but with increasing $V_{\text {rel }}$ a power law would not have been able to reproduce the change in curvature observed for the numerical data. Therefore, a function was needed which fulfilled the following constraints:

1. $C_{\text {rel }}\left(V_{\text {rel }}=1\right)=1$ and $C_{\text {rel }}\left(V_{\text {rel }}=V_{0}\right)=0$;

2. $d C_{\text {rel }} / d V_{\text {rel }}=0$ for $V_{\text {rel }}=V_{0}$;

3. $\left.\exists V_{\text {rel }} \epsilon\right] V_{0}, 1\left[\right.$ with $d^{2} C_{\text {rel }} / d v_{\text {rel }}^{2}=0$.

The function

$$
C_{\mathrm{rel}}\left(V_{\mathrm{rel}}\right)=a\left(\frac{V_{\mathrm{rel}}-V_{0}}{1-V_{0}}\right)^{3}+(1-a)\left(\frac{V_{\mathrm{rel}}-V_{0}}{1-V_{0}}\right)^{2}
$$

satisfies all of these constraints for $a<-0.5$. As the existence of local minima for $\left.V_{\text {rel }} \epsilon\right] V_{0}, 1$ [ would have been physically unrealistic, $a>-2$ was an additional requirement. For $a=1$ and $a=0$ Equation (10) would be an ordinary power law, but not fulfilling constraint 3 , as mentioned above. The parameter $a$, therefore, describes the difference between Equation (10) and a power law. $V_{0}$ contains the 
Table I. Scenario 1: Parameters used for fitting of numerical results

\begin{tabular}{lll}
\hline$\sigma$ & $a$ & $V_{0}$ \\
\hline 0.00 & -1.07 & 0.422 \\
0.10 & -1.28 & 0.513 \\
0.33 & -1.66 & 0.706 \\
0.50 & -1.83 & 0.815 \\
0.67 & -1.90 & 0.891 \\
1.00 & -1.94 & 0.970 \\
\hline
\end{tabular}

information, for which pore volume, and thus volume of biomass, the hydraulic conductivity reaches 0 .

Equation (10) was chosen to fit the numerical results with $a$ and $V_{0}$ as the fitting parameters. Best fits for $\sigma=1 / 3,1 / 2$ and $2 / 3$ were also shown in Figure 1. To monitor the sensitivity of the fitting parameters to $\sigma$ we also fitted numerical results using $\sigma=0,1 / 10$ and 1 (graphs not shown). The optimal values for $a$ and $V_{0}$ are given in Table I. For all values of $\sigma$ the numerical data could be fitted with a correlation coefficient of $R^{2}=0.998$. Both parameters show a significant correlation to $\sigma . V_{0}$, which is the relative pore volume at which $C_{\text {rel }}$ becomes 0 , increases with $\sigma$ and, therefore, the heterogeneity of the network, whereas $a$ decreases with $\sigma$.

\subsubsection{Calculation of Biomass Needed for Maximum Reduction of Hydraulic Conductivity}

From percolation theory it is known that if the density of open pores $p$ in an infinite network is below a critical value, called percolation threshold $p_{c}$, no percolation cluster exists. In such a case there is no continuous flow path and hydraulic conductivity is 0 . For $p>p_{c}$ a percolation cluster exists and hydraulic conductivity has a value greater than 0 . The value of $p_{c}$ depends on the dimensionality and the geometry of the pore network. For the network type used in this study $p_{c}$ has a value of 0.5 (Stauffer and Aharony, 1992).

For the present scenario it was assumed that the smallest pores are clogged first. Using this assumption it is possible to calculate the volume of biomass (and, therefore, the reduction of pore volume) needed to reduce the density of open respectively unclogged pores to a certain value $p$. Generally the volume $V$ of a given number of pores would be $V=\sum_{i} v\left(r_{i}\right)$. For infinite networks this can be replaced by $V=\int_{r} v(r) \xi(r) \mathrm{d} r$, which is the average volume per pore and where $\xi(r)$ is the probability distribution of the pore radius $r$. If $\xi$ is not expressed as a function of $r$ but instead as a function of $z=f(r)$ the pore volume can be expressed by

$$
V_{\mathrm{tot}}=\int_{z_{\min }}^{z_{\max }} v(z) \xi(z) \mathrm{d} z .
$$


In the case of a lognormal distribution of the pore radii the probability density function is

$$
\xi(z)=\frac{1}{\sqrt{2 \pi} \sigma} \exp \left(-\frac{1}{2} \frac{z^{2}}{\sigma^{2}}\right) \mathrm{d} z
$$

with $z=\ln \left(r / r_{a v}\right)$ and $r_{a v}$ as the average pore radius. With this and the assumption that clogging occurs in the smallest pore first Equation (11) can be transformed for $V_{\text {clogg }}$ as the volume of the clogged pores

$$
V_{\text {clogg }}=\sqrt{\frac{\pi}{2}} \frac{r_{a v}^{2} l}{\sigma} \int_{-\infty}^{z_{n}} \exp \left(-\frac{1}{2} \frac{z^{2}}{\sigma^{2}}+2 z\right) \mathrm{d} z
$$

where $z_{n}$ is the $z$-value corresponding to the fraction $n$ of pores clogged by the biomass.

The volume $V_{\text {bio }}$ of the clogged pores as a fraction of total pore volume can, therefore, be written as:

$$
V_{\text {bio }}=\frac{V_{\text {clogg }}}{V_{\text {tot }}}=\frac{1}{2}\left(1+\operatorname{erf}\left(\frac{z_{n}}{\sqrt{2} \sigma}-\sqrt{2} \sigma\right)\right)
$$

with erf $(x)$ as the error function.

To determine, which $z_{n}$ corresponds to a certain fraction $n$ of clogged pores the integral

$$
n=\int_{-\infty}^{z_{n}} \xi(x) \mathrm{d} x
$$

must be calculated. This equation can be solved for $z_{n}$ resulting in

$$
z_{n}=\sqrt{2} \sigma \operatorname{erf}^{-1}(2 n-1),
$$

where $\operatorname{erf}^{-1}$ is the inverse error function. With this Equation (14) can be transformed to

$$
V_{\text {bio }}=\frac{1}{2}\left(1+\operatorname{erf}\left(\operatorname{erf}^{-1}(2 n-1)-\sqrt{2} \sigma\right)\right)
$$

for the volume of biomass needed to clog a given fraction of pores of a pore network. To avoid percolation between the in- and outflow boundary of the network $n$ has to be equal to or larger than the percolation threshold $p_{c}$ (note that $n=1-p$ ).

All relations discussed are valid for infinite size pore networks. Whether the finite size networks used for the numerical simulation approximate them will be discussed later.

\subsubsection{Comparison of Theoretical and Numerical Results}

Figure 2 shows the volume of pores clogged by biomass as a function of the density of clogged pores $n$ (ratio of clogged pores to total number of pores) for the numerical simulations compared to the theoretical values (Equation (17)). It can be seen 


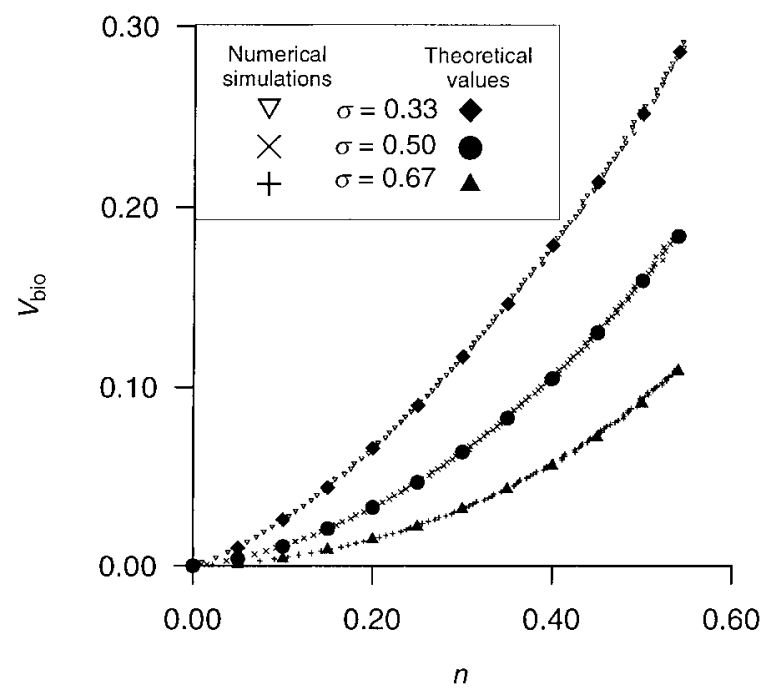

Figure 2. Comparison of numerically and theoretically calculated volumes of biomass as a function of density of clogged pores.

that these values are in good agreement. It can also be seen that even for $n>p_{c}$ data points from the numerical simulations exist. As only data for $C_{\text {rel }}>0$ are plotted, this contradicts the findings of percolation theory, which says that no percolation should be possible for $n>p_{c}$. This effect is caused by the finite size of the pore networks (e.g. Reynolds et al., 1980; Stauffer and Aharony, 1992). Figure 3 shows that increasing the size of the pore networks used for simulation yields a better approximation of the percolation threshold for infinite networks, $p_{c}=0.5$. For the

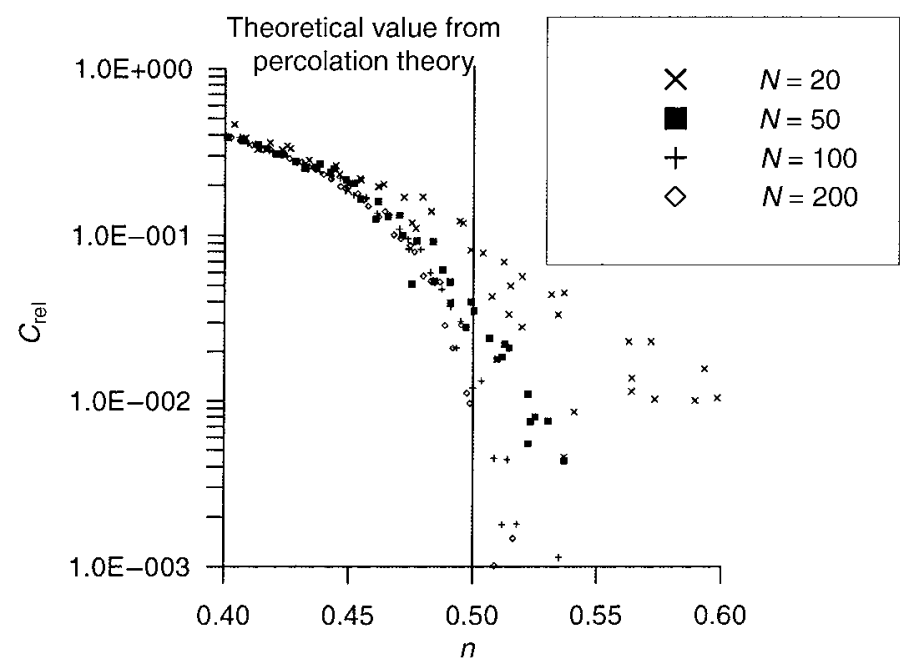

Figure 3. Hydraulic conductivity as a function of density of clogged pores, for different sizes of $N \times N$ pore networks. 
Table II. Scenario 1: Theoretically calculated volumes of biomass needed for maximal reduction of hydraulic conductivity

\begin{tabular}{llllll}
\hline$\sigma$ & \multicolumn{3}{c}{$n=p_{c}=0.5$} & & \multicolumn{2}{c}{$n=0.54$} \\
\cline { 2 - 3 } \cline { 5 - 6 } & $V_{\text {bio }}$ & $V_{\text {rel }}=1-V_{\text {bio }}$ & & $V_{\text {bio }}$ & $V_{\text {rel }}=1-V_{\text {bio }}$ \\
\hline 0.33 & 0.252 & 0.748 & 0.286 & 0.714 \\
0.50 & 0.159 & 0.841 & & 0.184 & 0.816 \\
0.67 & 0.091 & 0.909 & & 0.109 & 0.891 \\
\hline
\end{tabular}

$50 \times 50$ pore networks used in this study the threshold values for $n$ are about 0.54 (Figure 3). Volumes calculated with Equation (17) for the pore radii distribution used in this study are shown in Table II. It can be seen that the $V_{\text {rel-values for }}$ $n=p_{c}$ are larger than $V_{0}$ determined from the numerical simulations (Table I), but for $n=0.54$ the calculated values for $V_{\text {rel }}$ and $V_{0}$ correspond very well.

\subsection{SCENARIO 2: CLOGGING DUE TO A BIOFILM}

To investigate the influence of biofilm growth on hydraulic conductivity, simulations were done on $50 \times 50$ pore networks with lognormally distributed pore radii, in analogy to Scenario 1. As in Scenario 1, the standard deviations of the lognormal distributions were $\sigma=1 / 3,1 / 2$ and 2/3. The average pore radius, as defined in Section 3.1.2, was $r_{a v}=30 \mu \mathrm{m}$ and the pore length was $l=1.5 \mathrm{~mm}$ for all realizations. In each pore an initial biofilm thickness of $1 \mu \mathrm{m}$ was assumed. All other parameters used for the simulation (Table III) were adjusted to achieve the following effects.

Table III. Scenario 2: Parameters used for simulation of biofilm growth; masses are given in units of a reference mass $\mathrm{M}$

\begin{tabular}{lll}
\hline Parameter & Value & Description \\
\hline$\Delta H$ & $3 \cdot 10^{-2} \mathrm{~m}$ & $\begin{array}{l}\text { Piezometric head difference between } \\
\text { inflow and outflow } \\
\text { Inflow concentration of nutrient }\end{array}$ \\
$C_{\text {in }}$ & $1 \mathrm{Mm}^{-3}$ & $\begin{array}{l}\text { Rate constant for exchange between } \\
\text { exch }\end{array}$ \\
$7 \cdot 10^{-10} \mathrm{~ms}^{-1}$ & pore water and biofilm \\
$\mu^{+}$ & $10^{-4} \mathrm{~s}^{-1}$ & Rate constant for microbial growth \\
$\mu^{-}$ & $0.1 \mu^{+}$ & Rate constant for microbial decay \\
$Y$ & 0.1 & Yield factor \\
$z$ & $10^{-5} \mathrm{Mm}^{-3}$ & Rate constant for shear forces \\
$d$ & $10^{2} \mathrm{Mm}^{-3}$ & Density of biomass (dry mass per wet volume) \\
\hline
\end{tabular}


- The reduction of hydraulic conductivity of the entire pore network should be as high as possible.

- Biomass should not grow preferentially in the vicinity of the inflow of the pore networks.

The parameters $\Delta H$ and $z$ were controlling the extent of biofilm detachment by shear forces and high values of these parameters would have prevented the development of thick biofilm. $C_{\text {in }}$ and exch controlled the amounts of nutrient available to the microorganisms in the biofilm. A lower amount of nutrient supply would also have avoided the buildup of thick biofilms, whereas a higher value of exch would have caused high biomass production close to the inflow of the pore network, consuming nearly all of the nutrient already there. Parts of the pore network closer to its outflow would not have been supplied with the nutrient and no biomass growth would have taken place there. From the parameters $\mu^{+}, \mu^{-}$and $d, Y$, describing properties of the biomass, $d$ was the most important as it determined the biofilm thickness associated to the amount of biomass in a pore. Higher densities would have avoided bioclogging as the produced biomass would not have been able to change pore radii significantly. After the adjustment these values were kept constant for all simulations. For each $\sigma$, simulations were performed for 11 realizations. Numerical tests showed that single realization results did not change when the solution of the flow problem was only recalculated every 40th time step. For the results presented in this paper the flow field was recalculated after 20 simulation steps of transport and biofilm growth.

Results of these simulations are shown in Figure 4 for all values of $\sigma$. It can be seen that for all realizations a reduction in hydraulic conductivity of about two

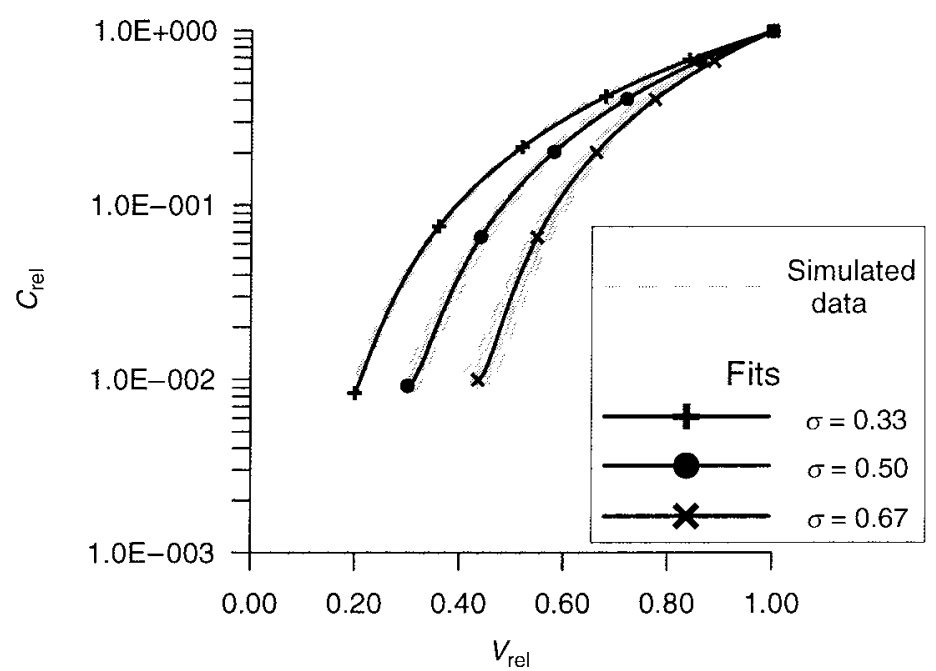

Figure 4. Reduction of hydraulic conductivity due to clogging of pores by a biofilm. Single realization results are compared to fits using Equation (18) with parameters given in Table IV. 
Table IV. Scenario 2: Parameters used for fitting of numerical results

\begin{tabular}{llll}
\hline$\sigma$ & $b$ & $C_{\text {rel }}^{\min }$ & $V_{0}$ \\
\hline 0.33 & $1.77 \pm 0.01$ & $(0.68 \pm 0.04) \times 10^{-2}$ & $0.178 \pm 0.004$ \\
0.50 & $1.78 \pm 0.02$ & $(0.93 \pm 0.06) \times 10^{-2}$ & $0.301 \pm 0.009$ \\
0.67 & $1.79 \pm 0.02$ & $(1.01 \pm 0.07) \times 10^{-2}$ & $0.436 \pm 0.014$ \\
\hline
\end{tabular}

orders of magnitude could be achieved. The amount of biomass needed to get a maximal clogging effect again depended on the heterogeneity of the pore radii distribution. The smaller $\sigma$ the more biomass (or pore volume reduction) was needed to reduce the hydraulic conductivity. Nevertheless, the simulation results indicated that the hydraulic conductivity was not dropping below a certain threshold and thus the pore network did not get completely clogged. Another observation was that with increasing $\sigma$ the differences between the $C_{\text {rel }}-V_{\text {rel }}$ curves for different single realizations, having the same $\sigma$, were increasing.

To express the numerical data analytically a power law could be used, which was only modified to take into account the existence of a lower (non zero) threshold $C_{\text {rel }}^{\min }$ for the relative hydraulic conductivity. Therefore, the function

$$
C_{\text {rel }}\left(V_{\text {rel }}\right)=\left(\left(\frac{V_{\text {rel }}-V_{0}}{1-V_{0}}\right)^{b}+C_{\text {rel }}^{\min }\right) \frac{1}{1+C_{\text {rel }}^{\min }}
$$

was used for fitting, with $V_{0}, C_{\text {rel }}^{\min }$ and $b$ as fitting parameters. In analogy to Scenario $1, V_{0}$ is the relative pore volume, for which the relative hydraulic conductivity reaches its minimum, and thus $1-V_{0}$ is the relative volume of biomass needed to reach the full clogging effect. To give the data points near the minimal $C_{\text {rel }}$ a higher weight, the $\log$-values of $C_{\text {rel }}$ were fitted. Fitting was done for each single realization and the resulting parameters were then averaged for every $\sigma$ (Table IV). It can be seen that $V_{0}$ and less strongly $C_{\text {rel }}^{\min }$ increase with increasing $\sigma$, whereas $b$ is nearly not affected by the degree of heterogeneity of the pore network. Figure 4 shows plots of Equation (18) using these averaged parameters in comparison to the single realization results.

To monitor the influence of the biofilm growth on the heterogeneity of the pore radii, one single realization was selected for each initial value of $\sigma$. The change of $\sigma$ of the pore radii distribution was monitored for different reductions of hydraulic conductivity (Figure 5). With increasing reduction of the hydraulic conductivity the pore radii became more heterogeneous until the highest values for $\sigma$ were reached at $C_{\text {rel }} \approx 0.1$. Then the heterogeneity decreased again slightly, but remained clearly higher than for the unclogged networks. 


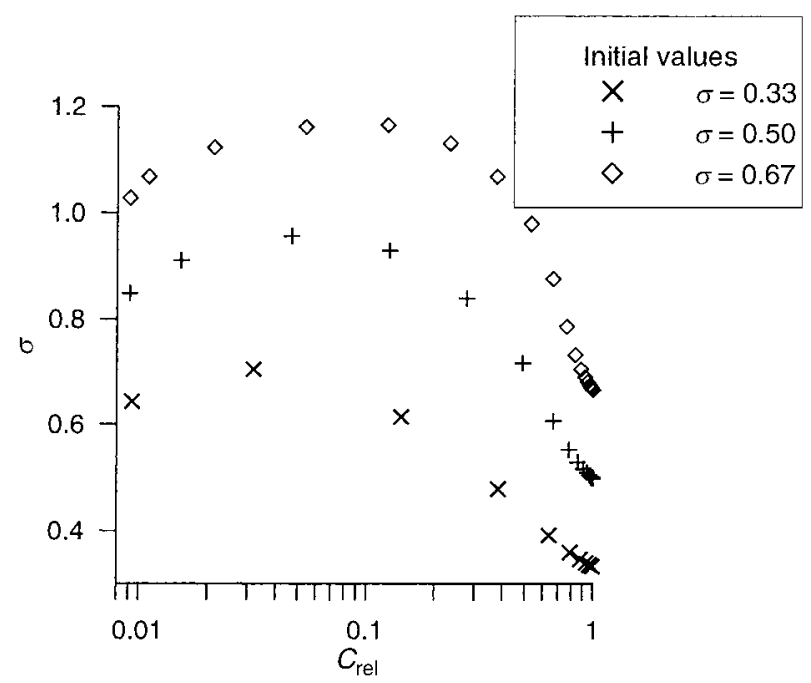

Figure 5. Change of heterogeneity of pore radii distribution for single pore networks clogged by a biofilm.

\section{Discussion}

Using pore network models to investigate the effects of bioclogging on a pore scale for different scenarios of microbial growth shows that the change of hydraulic properties of the entire pore network strongly depends on the morphology of microbial growth in the pores.

\subsection{CLOGGING DUE TO MICROBIAL COLONIES}

In Scenario 1 where the biomass was assumed to be present in form of microbial colonies, which occupy the smallest pores of the network first, it was possible to simulate reductions of hydraulic conductivity of more than two orders of magnitude. The amount of biomass needed to achieve a certain reduction of hydraulic conductivity strongly depended on the heterogeneity of the pore network. The higher the heterogeneity (within this study expressed by the standard deviation of the lognormal pore radii distribution) the less biomass was needed to clog the entire network. A function was presented, which could be successfully fitted to the numerical data by adjusting two independent parameters $V_{0}$ and $a$ only. $V_{0}$ is interpreted as the relative pore volume for which the hydraulic conductivity reaches 0 , and thus $1-V_{0}$ is the relative volume of biomass needed to clog the entire network. The parameter $a$ quantifies the difference between Equation (10) and a power law. In addition, $a$ contains the information how the hydraulic conductivity decreases initially for small changes of pore volume. The slope of this initial decrease $\left(d C_{\text {rel }} / d V_{\text {rel }}\right)$ is $(a+2) /\left(1-V_{0}\right)$ for $V_{\text {rel }}=1$. For homogeneous networks $a$ was close to -1 and thus this slope was approximately $1 /\left(1-V_{0}\right)$, 
which would be the slope when expressing a linear decrease between $C_{\text {rel }}=1$ at $V_{\text {rel }}=1$ and $C_{\text {rel }}=0$ at $V_{\text {rel }}=V_{0}$. With increasing heterogeneity, $a$ was approached -2 , which corresponded to a slope of 0 . This means that for homogeneous networks the removal of a small number of pores contributes equally to the decrease in $C_{\text {rel }}$ and $V_{\text {rel }}$, whereas for heterogeneous networks removing a small number of pores almost does not change $C_{\text {rel }}$. This behaviour is obviously determined by the way the pores are selected for removal. In this study the smallest pores were plugged first, and thus a removal of only a small number of pores effected $C_{\text {rel }}$ less than $V_{\text {rel }}$ (note that the hydraulic conductivity of a single pore $i$ scales with $r_{i}^{4}$, whereas its volume scales with $r_{i}^{2}$ ), especially for high degrees of heterogeneity. As a consequence $a$ is not only affected by the heterogeneity of the pore radii but also by the way pore plugging and pore radius are correlated. A preferential clogging of the smallest pores results in values of $a$ closer to -2 , and a weaker correlation between pore radii and clogging probability would result in values of $a$ closer to -1 .

For practical reasons one can summarize that the key parameter $V_{0}$ is given by the minimum of the $C_{\text {rel }}-V_{\text {rel }}$ curves. To determine $1-V_{0}$, which is the volume of biomass needed to get the maximal clogging effect, it is possible to use results from percolation theory. For a large variety of network geometries percolation thresholds are known (Sahimi, 1995) and if in addition the pore size distribution is known, Equation (17) provides the possibility to predict the volume of biomass necessary to clog these networks. If $V_{0}$ is known, the parameter $a$ can be estimated from the slope of the $C_{\text {rel }}-V_{\text {rel }}$ curve for $V_{\text {rel }}$ close to 1 .

\subsection{Clogging DUe to A Biofilm}

In Scenario 2 it was assumed that biomass grows as a biofilm at the walls of each pore. Results of the simulations could show that this biofilm was able to reduce the hydraulic conductivity of the entire network by up to two orders of magnitude. For this scenario it was possible to find an analytical expression linking hydraulic conductivity and pore volume using a power law, which could be fitted to the numerical results successfully. Parameters determined by this procedure again show the importance of determination of $V_{0}$ as the parameter varying most sensitively with $\sigma$. The exponent $b$ of the used power law seems to be independent of the heterogeneity of the pore network. Whether this is a general behaviour for networks with biofilm growth can only be answered by further studies. For the upscaling of pore network results to the scale of a representative elementary volume this question is of great importance. Simulation results of this study indicate that biofilm growth is increasing the heterogeneity of a network's pore radii distribution. If these results can be confirmed by further investigations (numerical simulations or preferably experimental studies), they would contradict assumptions made by Clement et al. (1996), where preferential clogging of the larger pores was assumed, which led to higher volumes of biomass needed to reduce the hydraulic conductivity. 


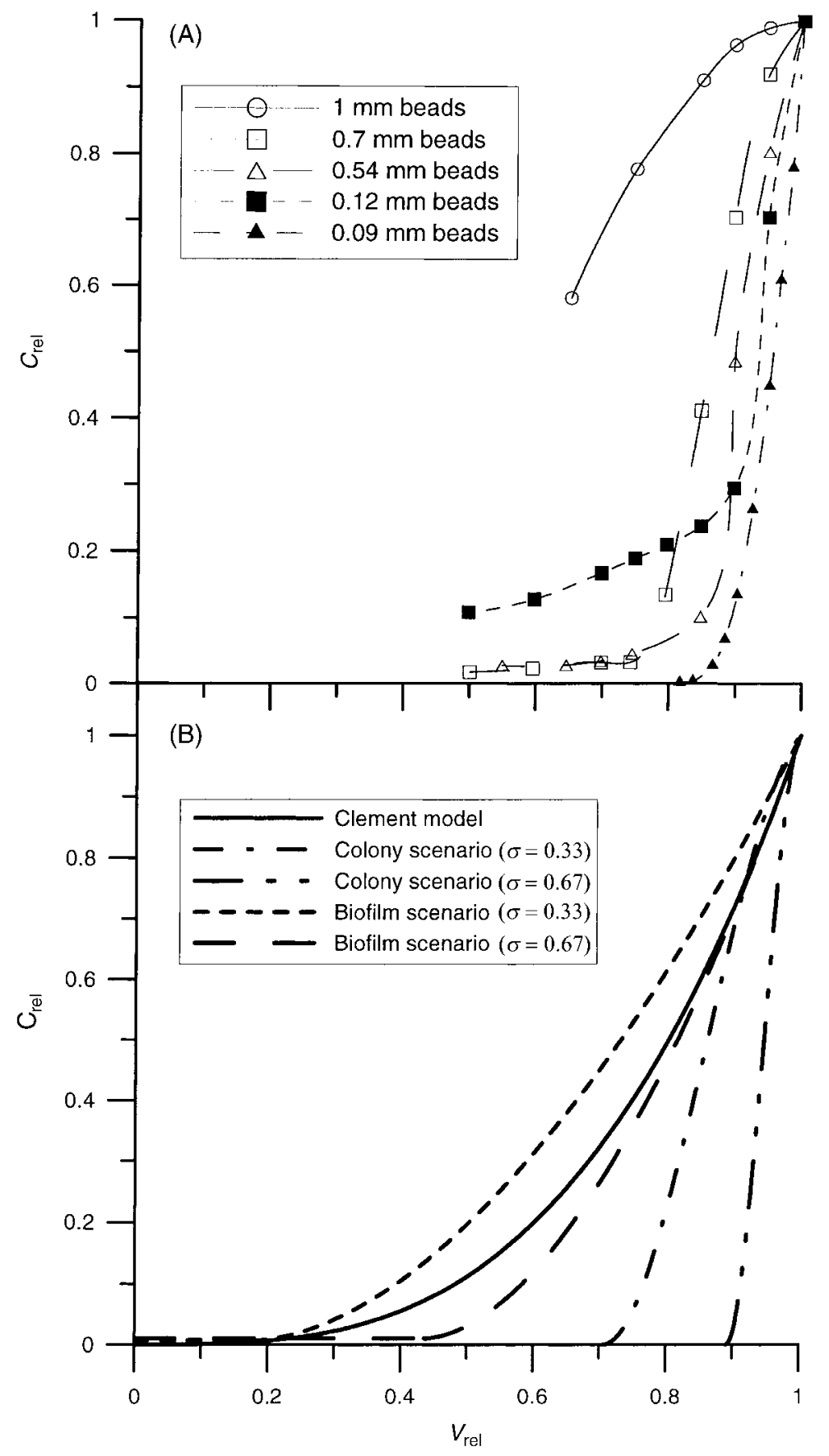

Figure 6. Comparison of measured (A) and predicted (B) reductions of hydraulic conductivity. Measured data were taken from Vandevivere et al. (1995), the Clement model is taken from Clement et al. (1996). 


\subsection{COMPARISON BETWEEN NUMERICAL AND EXPERIMENTAL RESULTS}

Comparing the results of both scenarios to experimental results reviewed in Vandevivere et al. (1995) and to the clogging model of Clement et al. (1996) (Figure 6), the most significant difference is the different amount of biomass volume needed to reduce the hydraulic conductivity of the entire network. Microbial colonies are much more efficient in clogging a pore network than a continuous biofilm. The reduction of hydraulic conductivity derived for the colony scenario is similar to hydraulic conductivity reductions measured for porous media with grain sizes of less than $1 \mathrm{~mm}$. The fact that for the presented experiments hydraulic conductivity was not reduced to 0 but maintained a small residual conductivity can not be explained with results from the colony scenario. Assuming that pores completely filled with biomass retain a very small but finite hydraulic conductivity, a residual conductivity can be reproduced within the colony scenario, too.

The existence of a residual hydraulic conductivity can be explained using the biofilm scenario, but the general decrease in hydraulic conductivity is strongly underpredicted by the biofilm scenario for grain sizes below $1 \mathrm{~mm}$. In contrast, the reduction of hydraulic conductivity for a grain size of $1 \mathrm{~mm}$ is only slightly overpredicted by the biofilm scenario. This is at least an indication that for porous media with grain sizes below $1 \mathrm{~mm}$, the assumption is more likely that the biomass is growing in colonies and not in a biofilm. For a porous medium made of $1 \mathrm{~mm}$ beads the measured decrease in hydraulic conductivity was less severe than for smaller grain sizes and predictions assuming a biofilm growth in the pores are better suited to describe the clogging for this bigger grain size. This would indicate that the growth morphology changes from colony growth to biofilm growth with increasing grain sizes and increasing average pore radii. As the formation of large plugs, able to clog pores with large radii, is less likely than the formation of small plugs, the assumption is reasonable that porous media made of coarse grains can not be clogged by microbial colonies.

Results for the biofilm scenario were similar to predictions of the model of Clement et al. (1996). Although in Clement et al. (1996) no assumptions were made according to the distribution of biomass in the pores, it was shown in their study that their model predictions were very similar to results of the biofilm model of Taylor et al. (1990), which was derived for bundles of non-interconnected pores. This indicates that in case of porous media clogged by a biofilm the pore interconnectivity has only small influence on the clogging effects.

The initial heterogeneity of the pore radii distribution had the same effect in both scenarios. The higher the initial heterogeneity the less biomass was needed to clog the entire network. The influence of biomass itself on the pore radii distribution differed between the two scenarios. For the colony scenario small pores were clogged preferentially and thus the heterogeneity of the remaining unclogged pores was reduced due to the bioclogging. In contrast it was shown in this study that for the biofilm scenario the heterogeneity of the pore radii distribution was increased due to bioclogging. 


\subsection{LIMITATION OF PORE NETWORK RESULTS}

Results of this study were based on simplified assumptions on the growth of biomass in the pore space and the geometry of the used pore networks was also a simplification of a real porous medium. It must be assumed that this may affect the $C_{\text {rel }}-V_{\text {rel }}$ relations derived from the pore network simulations. The hydraulic properties of pore networks mainly depend on the coordination number $Z$ (number of pores per node) (Jerauld et al., 1984a, b) and thus increasing the dimensionality of the rectangular network used in this study from two to three would increase its coordination number from $Z=4$ to $Z=6$. This increases the degrees of freedom for the water to flow through the network and it is reasonable to assume that a network can be clogged less easily then. The fact that the percolation threshold is getting smaller with increasing coordination number supports this assumption. Nevertheless, this does not necessarily mean that natural porous media have a higher coordination number than the pore networks used in this study. Sahimi (1995) reported that the average coordination number for sandstones was found to be between 4 and 8, whereas Vogel and Roth (1998) found an average coordination number of approximately 2.5 for the A-and B-horizon of a forest soil. For the colony scenario we present a function (Equation (17)), which links the change in $V_{0}$ to the geometry and coordination number of the network. As Equation (17) is only limited by assumptions regarding the biomass distribution, it can be used to estimate the volume of biomass needed to clog a given network. Only the knowledge of the percolation threshold and the pore size distribution of the network is required. To determine, which type of network is the best representation of a natural porous medium, several techniques are available. Techniques for investigating porous media structures are, for example, mercury injection, electron microscopy or NMR; a method transferring the topology of a natural soil, analyzed by image analysis of serial sections, into a pore network model is, for example, described in Vogel and Roth (1998). In this study we did not assume a spatial correlation of the pore radii. In case of porous media with spatial correlation lengths larger than the length of a pore, preferential flow may occur within the pore network. Especially for the colony scenario this would reduce the ability of the biomass to clog the entire network, as preferential flow paths contain mainly large pores. For the biofilm scenario the occurrence of preferential flow paths may have less influence on the clogging effects.

Another aspect may influence the results derived from pore network simulations in general. In this study the sites connecting the pores of the pore network were assumed to have no volume. If they contributed significantly to the total pore volume of the network, the $C_{\text {rel }}-V_{\text {rel }}$ relations would be different. Assuming that the pressure loss along these sites would be negligible, the biomass would have a higher clogging efficiency and thus the maximum reduction of hydraulic conductivity would be reached for smaller reductions of porosity. In addition it must be pointed out that simulations for the biofilm scenario were done for constant head boundary conditions. Therefore, the flow velocity in the pores was decreasing with 
decreasing $C_{\text {rel }}$, and thus biofilm detachment by shear forces was not a dominant process. Assuming constant flux boundaries would cause the flow velocity in the pores to increase with increasing biofilm thickness. In this case biofilm detachment by shear forces as well as the transport and reattachment of the detached biomass would become an important process for the evolution of the clogging effects.

The absolute values of the parameters obtained in this study may not be representative for microbial growth in a natural porous medium (e.g. sand). Nevertheless, the general functional relationships provide an explanation for measured data such as those shown in Figure 6. For a further confirmation of the validity of the concept more experiments are necessary. Such experiments may be performed on a lab scale or on artificial pore networks (e.g. Wilson, 1996; Dupin and McCarty, 1999, 2000; Kim and Fogler, 2000).

\section{Conclusion}

Pore network simulations performed in this study could show that the high reductions in hydraulic conductivity of porous media, which for grain sizes below $1 \mathrm{~mm}$ were observed already for small reductions in porosity (Vandevivere et al., 1995), can be explained theoretically by assuming a colony scenario for microbial growth. In contrast, assuming microbial growth in a biofilm was underpredicting the experimentally observed clogging effects, except of those of a porous medium with a grain size of $1 \mathrm{~mm}$. This indicates that the assumption of microbial colonies causing the bioclogging is more likely for porous media with grain sizes below $1 \mathrm{~mm}$.

$V_{0}$ is the key parameter for both scenarios. It may depend on the geometry of the pore networks. At least for the colony model a function was presented in this study, which described the dependence of $V_{0}$ on the geometry and heterogeneity of the pore network.

\section{Acknowledgements}

The authors like to thank Geri Wagner, Israel Institute of Technology, Haifa, Israel for his support. This study was funded by the Swiss Federal Institute of Technology (ETH) Zurich, Switzerland.

\section{References}

Baveye, P. and Dumestre, A.: 1998, Comments on: experimental study on the reduction of soil hydraulic conductivity by enhanced biomass growth by Wu et al., Soil Sci. 163(9), 759-761.

Baveye, P., Vandevivre, P., Hoyle, B. L., DeLeo, P. C. and Sanchez de Lozada, D.: 1998, Environmental impact and mechanisms of the biological clogging of saturated soils and aquifer materials, Crit. Rev. Environ. Sci. Technol. 28(2), 123-191.

Berkowitz, B. and Balberg, I.: 1993, Percolation theory and its application to groundwater hydrology, Water Resour. Res. 29(4), 775-794. 
Brough, M. J., Al-Tabbaa, A. and Martin, R. J.: 1997, Active biofilm barriers for waste containment and bioremediation: laboratory assessment, in: Proc. Int. Symp. In Situ and On-site Bioremediation, New Orleans, USA, May, Vol. 4, pp. 233-238.

Brun, A., Engesgaard, P., and Frind, E. O.: 1994, A coupled microbiology-geochemistry transport model for saturated groundwater flow, in: T. Dracos and F. Stauffer (eds), IAHR/AIRH Symposium on Transport and Reactive Processes in Aquifers, Zurich, Switzerland, A. A. Balkema, Rotterdam, The Netherlands, pp. 457-462.

Brutsaert, W.: 1966, Probability laws for pore-size distributions, Soil Sci. 101(2), 85-92.

Burganos, V. N., Paraskeva, C. A. and Payatakes, A. C.: 1995, Monte-Carlo network simulation of horizontal, upflow and downflow depth filtration, AICHE J. 41(2), 272-285.

Chang, F. F. and Civan, F.: 1997, Practical model for chemically induced formation damage, J. Petrol. Sci. Engng 17, 123-137.

Characklis, W. G. and Marshall, K. C. (eds): 1990, Biofilms, Wiley, New York.

Chiang, C. Y., Dawson, C. N. and Wheeler, M. F.: 1991, Modeling of in situ biorestoration of organic compounds in groundwater, Transport in Porous Media 6, 667-702.

Clement, T. P., Hooker, B. S. and Skeen, R. S.: 1996, Macroscopic models for predicting changes in saturated porous media properties caused by microbial growth, Ground Water 34(5), 934942.

Coskuner, G.: 1997, Microvisual study of multiphase gas condensate flow in porous media, Transport in Porous Media 28(1), 1-18.

Cunningham, A. B., Charaklis, W. G., Abedeen, F. and Crawford, D.: 1991, Influence of biofilm accumulation on porous media hydrodynamics, Environ. Sci. Technol. 25(7), 1305-1311.

Dupin, H. J. and McCarty, P. L.: 1999, Mesoscale and microscale observations of biological growth in a silicon pore imaging element, Environ. Sci. Technol. 33(8), 1230-1236.

Dupin, H. J. and McCarty, P. L.: 2000, Impact of colony morphologies and disinfection on biological clogging in porous media, Environ. Sci. Technol. 34(8), 1513-1520.

Ewing, R. P. and Gupta, S. C.: 1994, Pore-scale network modeling of compaction and filtration during surface sealing, Soil Sci. Soc. Amer. J. 58(3), 712-720.

Imdakm, A. O. and Sahimi, M.: 1991, Computer simulation of particle transport processes in flow through porous media, Chem. Engng Sci. 46(8), 1977-1993.

Jerauld, G. R., Hatfield, J. C., Scriven, L. E. and Davis, H. T.: 1984a, Percolation and conduction on Voronoi and triangular networks: a case study in topological disorder, J. Phy. C-Solid State Phy. 17, 1519-1529.

Jerauld, G. R., Scriven, L. E. and Davis, H. T.: 1984b, Percolation and conduction on the 3D Voronoi and rectangular networks: a second case study in topological disorder, J. Phy. C-Solid State Phy. 17, 3429-3439.

Johnston, C. D., Rayner, J. L., De Zoysa, D. S., Ragusa, S. R., Trefry, M. G. and Davis, G. B.: 1997, Studies of bioclogging for containment and remediation of organic contaminants, in: Proc. Int. Symp. In Situ and On-site Bioremediation, New Orleans, USA, May, Vol. 4, pp. 241-246.

Kaiser, C.: 1997, A direct percolation model for clogging in a porous medium with small Inhomogeneities, Transport in Porous Media 26, 133-146.

Kim, D.-S. and Fogler, H. S.: 2000, Biomass evolution in porous media and its effects on permeability under starvation conditions, Biotechnol. Bioengng 69(1), 47-56.

Kindred, J. S. and Celia, M. A.: 1989, Contaminant transport and biodegradation. 2. Conceptual model and test simulation, Water Resour. Res. 25, 1149-1160.

Kinzelbach, W., Schäfer, W. and Herzer, J.: 1991, Numercal modeling of natural and enhanced denitrification processes in aquifers, Water Resour. Res. 27, 1123-1135.

Lee, J. and Koplik, J.: 2001, Newwork model for deep bed filtration, Phy. Fluids 13(5), 10761086.

Lensing, H. J., Vogt, M. and Herrling, B.: 1994, Modeling of biological mediated redox processes in the subsurface, J. Hydrol. 159, 125-143. 
Loehle, C. and Johnson, P.: 1994, A framework for modeling microbial transport and dynamics in the subsurface, Ecol. Model. 73, 31-49.

MacQuarrie, K. T. B., Sudicky, E. A. and Frind, E. O.: 1990, Simulation of biodegradable organic contaminants in groundwater. 1. Numerical formulation in principal directions, Water Resour. Res. 26, 207-222.

Monod, J.: 1942, Recherce sur la croissance des cultures bactérienne, in: J. Bordet (ed.), Actualités scientifiques et industrielle, Vol. 911 of Microbiologie, Hermann \& $\mathrm{C}^{\mathrm{ie}}$, Paris, France, pp. 1-121.

Rege, S. D. and Fogler, H. S.: 1987, Network model for straining dominated particle entrapment in porous media, Chem. Engng Sci. 42(7), 1553-1564.

Rege, S. D. and Fogler, H. S.: 1991, Development of radial models for formation damage in porous media, Chem. Engng Commun. 108, 67-83.

Reynolds, P. J., Stanley, H. E. and Klein, W.: 1980, Large-cell Monte Carlo renomalization group for percolation, Phy. Rev. B 21(3), 1223-1245.

Sahimi, M.: 1995, Flow and Transport in Porous Media and Fractured Rock, VCH Verlagsgesellschaft, Weinheim, Germany.

Schäfer, D., Schäfer, W. and Kinzelbach, W.: 1998, Simulation of reactive processes related to biodegradation in aquifers. 1. Structure of the three-dimensional reactive transport model, J. Contam. Hydrol. 31(1-2), 167-186.

Stauffer, D. and Aharony, A.: 1992, Introduction in Percolation Theory, revised 2nd edn, Taylor \& Francis Group, Basingstoke Haunts, U.K.

Suchomel, B. J., Chen, B. M. and Allen, M. B.: 1998a, Macroscale properties of porous medium from a network model of biofilm processes, Transport in Porous Media 31, 39-66.

Suchomel, B. J., Chen, B. M. and Allen, M. B.: 1998b, Network model of flow, transport and biofilm effects in porous media, Transport in Porous Media 30, 1-23.

Sugita, F., Gillham, R. W. and Mase, C.: 1995, Pore scale variation in retardation factor as a cause of nonideal reactive breakthrough curves. 2. Pore network analysis, Water Resour. Res. 31(1), $113-119$

Taylor, S. W., Milly, P. C. D. and Jaffé, P. R.: 1990, Biofilm growth and the related changes in the physical properties of a porous medium. 2. Permeability, Water Resour. Res. 26(9), 21612169.

Taylor, S. W. and Jaffé, P. R.: 1990a, Biofilm growth and the related changes in the physical properties of a porous medium. 1. Experimental investigations, Water Resour. Res. 26(9), 2153-2159.

Taylor, S. W. and Jaffé, P. R.: 1990b, Biofilm growth and the related changes in the physical properties of a porous medium. 3. Dispersivity and model verification, Water Resour. Res. 26(9), 21712180.

Tien, C. and Payatakes, A. C.: 1979, Advance in deep bed filtration, AICHE J. 25, 737-759.

Vandevivere, P.: 1995, Bacterial clogging of porous media: a new modelling approach, Biofouling 8 , 281-291.

Vandevivere, P. and Baveye, P.: 1992, Saturated hydraulic conductivity reduction caused by aerobic bacteria in sand columns, Soil Sci. Soc. Amer. J. 56(1), 1-13.

Vandevivere, P., Baveye, P., Sanchez de Lozada, D. and DeLeo, P.: 1995, Microbial clogging of saturated soils and aquifer materials: evaluation of mathematical models, Water Resour. Res. 31(9), 2173-2180.

Vogel, H. J.: 1997, Morphological determination of pore connectivity as a function of pore size using serial sections, Eur. J. Soil Sci. 48, 365-377.

Vogel, H. J. and Roth, K.: 1998, A new approach for determining effective soil hydraulic functions, Eur. J. Soil Sci. 49, 547-556.

Wang, X. and Mohanty, K. K.: 1999, Critical condensate saturation in porous medium, J. Coll. Inter. Sci. 214, 416-426. 
Widdowson, M. A., Molz, F. J. and Benefield, L. D.: 1988, A numerical transport model for oxygenand nitrate-based respiration linked to substrate and nutrient availability in porous media, Water Resour. Res. 24, 1553-1565.

Wilson, J. L.: 1996, Visualization experiments at the pore scale, in: K. Kobus, B. Barczewski and H.-P. Koschitzky (eds), Groundwater and Subsurface Remediation, Springer, Berlin/Heidelberg.

Wojtanowicz, A. K., Krilov, Z. and Langlinais, J. P.: 1988, Experimental determination of formation damage pore blocking mechanisms, Transact. ASME 110, 34-42.

Wu, J., Giu, S., Stahl, P. and Zhang, R.: 1997, Experimental study on the reduction of soil hydraulic conductivity by enhanced biomass growth, Soil Sci. 162(10), 741-748. 